\title{
Photon-induced near-field electron microscopy: Mathematical formulation of the relation between the experimental observables and the optically driven charge density of nanoparticles
}

\author{
Sang Tae Park and Ahmed H. Zewail, \\ Physical Biology Center for Ultrafast Science and Technology, Arthur Amos Noyes Laboratory of Chemical Physics, \\ California Institute of Technology, Pasadena, California 91125, USA
}

(Received 17 September 2013; revised manuscript received 16 October 2013; published 31 January 2014)

\begin{abstract}
Photon-induced near-field electron microscopy (PINEM) enables the visualization of the plasmon fields of nanoparticles via measurement of photon-electron interaction [S. T. Park et al., New J. Phys. 12, 123028 (2010)]. In this paper, the field integral, which is a mechanical work performed on a fast electron by the total electric field, plays a key role in understanding the interaction. Here, we reexamine the field integral and give the physical meaning by decomposing the contribution of the field from the charge-density distribution. It is found that the "near-field integral" (the near-field approximation of the field integral) can be expressed as a convolution of the two-dimensional projection of the optically driven charge-density distribution in the nanoparticle with a broad radial response function. This approach, which we call the "convolution method," is validated by applying it to Rayleigh scattering cases, where previous analytical expressions for the field integrals in near-field approximations are reproduced by the convolution method. The convolution method is applied to discrete dipole approximation calculations of a silver nanorod, and the nature of the induced charge-density distributions of its plasmons is discussed.
\end{abstract}

DOI: 10.1103/PhysRevA.89.013851

PACS number(s): 73.20.Mf, 78.67.Qa, 34.50.Bw

\section{INTRODUCTION}

Plasmons dictate the optical responses of metals in the visible range, and localized surface plasmons (LSP) in metallic nanoparticles exhibit a subwavelength confinement of electromagnetic waves and field enhancement [1-3]. Hence, LSP plays an important role in plasmonics, subdiffraction wave guides, and chemical sensing. For example, surface-enhanced Raman scattering (SERS) can exhibit an enhancement of $10^{8}-$ $10^{14}$ by utilizing plasmon resonance, making single-molecule detection possible [4,5]. However, plasmon excitation and field enhancement in nanoparticles are highly sensitive to the geometry of the particle, and understanding their relation to structure is of great importance in developing those fields. Thus, experimental methods to probe the plasmon field enhancement are indispensable in understanding the phenomenon and advancing plasmon studies.

Nelayah et al. invoked spectral imaging (SI) to "map" plasmons in a nanoprism using scanning transmission electron microscopy and electron-energy-loss spectroscopy (STEMEELS) and resolved three different plasmon modes [6,7]. It was also demonstrated that energy-filtered transmission electron microscopy (EFTEM) can be equivalently employed to obtain the same information [8]. Plasmons in nanoparticles of other geometries were also visualized with SI methods [9-23]. In particular, to investigate SERS activity, Guiton et al. employed STEM-EELS to probe the fields of plasmons in silver nanorods and correlated this with far-field scattering spectroscopy [15]. They further investigated EELS with a discrete dipole approximation (DDA) simulation [24] and compared it to the optical excitation, in particular for the junction field [25]. Rossouw et al. studied a longer nanorod and showed that the polarization can be described as standing waves in a Fabry-Perot resonator [26].

\footnotetext{
*zewail@ caltech.edu
}

In addition to EELS, García de Abajo and Kociak proposed electron-energy-gain spectroscopy (EEGS) [27], which allows electron spectroscopy with an energy resolution determined by the laser bandwidth, by exciting a plasmon mode in a nanoparticle with an external light and probing it via the energy-gain process of a swift electron. Barwick et al. demonstrated a time-resolved imaging technique for nanostructures with a femtosecond laser in ultrafast electron microscopy (UEM) and termed it photon-induced near-field electron microscopy (PINEM), emphasizing the capability to image the plasmon fields $[28,29]$. Theoretical accounts have been given by various groups [30-33]. In particular, the PINEM (image) intensity is approximately proportional to the absolute square of the "field integral," which is defined as the mechanical work performed on the moving electron by the scattered electromagnetic wave (electric field) [34]. Efforts have been made to correlate the PINEM image to the electric-field distribution itself [31,35-38].

Here, we show that PINEM can be mathematically related to the optically driven charge-density distributions of nanoparticle plasmons. Namely, it is found that the field integral (the interaction term) can be expressed as a convolution of the induced charge-density projection, with a broad radial response function, when we recognize that the near field can be expressed as the Coulomb field of the instantaneous (oscillating) charge density. We demonstrate that the induced charge-density picture provides a more direct interpretation of PINEM than the electric fields or the polarizations of plasmons.

\section{THEORY}

In order to understand PINEM images, we first describe the theory of photon-electron interaction and define the field integral, which is expressed with the scattered electric field. We invoke Maxwell's equations to relate the near field of the light scattering to the induced charge density and then evaluate the 
field integral in terms of charge density, which we will show provides physical insight.

\section{A. Photon-electron coupling}

Detailed derivations of the transition probabilities of PINEM in nonrelativistic and relativistic formulations were given in Refs. [31] and [34], respectively, where the timedependent one-dimensional (1D) Schrödinger or Dirac equation of a free electron under a scattered electromagnetic wave was analytically solved to first order. In those semiclassical treatments of PINEM, however, it was assumed that the scalar potential vanishes while using Coulomb gauge, which may not be the case. Here, we reexamine the theoretical formulation of PINEM for a nonvanishing scalar potential.

The Hamiltonian of an electron under electromagnetic interaction is given by

$$
\hat{H}=\frac{1}{2 m}(\hat{\mathbf{p}}-q \mathbf{A})^{2}+q V,
$$

where $q=-e$ is the electron charge, $m$ is the electron mass, $V$ is the scalar potential, and $\mathbf{A}$ is the vector potential (see Sec. II B).

The wave function of the electron is defined in terms of the envelope function $f(z, t)$ such that $\Psi(z, t)=$ $f(z, t) \exp \left[i\left(k_{c} z-\omega_{c} t\right)\right]$, where $k_{c}$ and $\omega_{c}$ are chosen to correspond to the actual (relativistic) velocity $v_{0}$ such that $\hbar k_{c}=m v_{0}$ and $\hbar \omega_{c}=\frac{1}{2} m v_{0}^{2}$. The envelope function carries the initial wave-packet profile and the final phase change due to the interaction with the scattered electric field. Using the Coulomb gauge, such that $\boldsymbol{\nabla} \cdot \mathbf{A}=0$, with a nonvanishing scalar potential, the time-dependent one-dimensional Schrödinger equation becomes

$$
\begin{aligned}
& \frac{\partial f}{\partial t}+\frac{\hbar k_{c}}{m} \frac{\partial f}{\partial z}-\frac{i \hbar}{2 m} \frac{\partial^{2} f}{\partial z^{2}} \\
& =+i \frac{k_{c} q}{m} A_{z} f+\frac{q}{m} A_{z} \frac{\partial f}{\partial z}+\frac{q^{2} A_{z}^{2}}{2 i \hbar m} f+\frac{q V}{i \hbar} f,
\end{aligned}
$$

where the second and third terms on the left-hand side are the propagation and dispersion terms, respectively. Ignoring the dispersion and the ponderomotive term, we obtain

$$
\begin{aligned}
& \frac{\partial f(z)}{\partial t}+\frac{\hbar k_{c}}{m} \frac{\partial f(z)}{\partial z} \\
& \approx+i \frac{k_{c} q}{m} A_{z}(z, t) f(z)+\frac{q}{i \hbar} V(z, t) f(z) .
\end{aligned}
$$

It is convenient to redefine the envelope function on the moving frame $g\left(z^{\prime}, t^{\prime}\right)$ such that $f(z, t)=g\left(z-v_{0} t, t\right)$. The moving frame, $z^{\prime}=z-v_{0} t$, is chosen to be at the same velocity as the group velocity of the electron packet, using $\frac{\hbar k_{c}}{m}=v_{0}$, such that the propagation term is canceled. Defining $U(z, t)=V(z, t)-$ $v_{0} A_{z}(z, t)$, Eq. (3) reduces to

$$
\frac{\partial g\left(z^{\prime}\right)}{\partial t} \approx-i \frac{q}{\hbar} U\left(z^{\prime}+v_{0} t, t\right) g\left(z^{\prime}\right),
$$

which is an ordinary differential equation with the solution given by

$$
g\left(z^{\prime}, t\right)=g\left(z^{\prime}, t_{0}\right) \exp \left[-i \frac{q}{\hbar} \int_{t_{0}}^{t} d t^{\prime} U\left(z^{\prime}+v_{0} t^{\prime}, t^{\prime}\right)\right],
$$

and the final solution is obtained at $t \rightarrow+\infty$ for $t_{0} \rightarrow-\infty$. $U(z, t)$ in Eq. (5) is further approximated by invoking a slowly varying envelope approximation to factor out the temporal oscillation and the incident light intensity profile as

$$
U(z, t) \approx \operatorname{Re}\left\{\tilde{U}_{0}(z, 0) e^{-i \omega_{p} t} e^{-\frac{(t+\tau)^{2}}{4 \sigma_{p}^{2}}}\right\},
$$

where $\omega_{p}$ is the optical angular frequency, $\sigma_{p}$ is the incident light duration, $\tau$ is the time delay between the photon and the electron, and we ignored the propagation of the scattered light and assumed that the potentials (and electric field) are linearly proportional to the electric field of the incident light at the scattering center $(z=0)$, which is given by the Gaussian profile with $\sigma_{p}$ and $\tau$. With $z^{\prime \prime} \equiv z^{\prime}+v_{0} t^{\prime}$ and $\Delta k \equiv \frac{\omega_{p}}{v_{0}}$, the final solution becomes

$$
\begin{aligned}
\frac{g\left(z^{\prime},+\infty\right)}{g\left(z^{\prime},-\infty\right)} & =\exp \left[-i \frac{q}{\hbar} \int_{-\infty}^{+\infty} \frac{d z^{\prime \prime}}{v_{0}} \operatorname{Re}\left\{\tilde{U}\left(z^{\prime \prime}, 0\right) e^{-i \Delta k\left(z^{\prime \prime}-z^{\prime}\right)} e^{-\frac{\left(z^{\prime \prime}-z^{\prime}+v_{0} \tau\right)^{2}}{4 v_{0}^{2} \sigma_{p}^{2}}}\right\}\right] \\
& \approx \exp \left[-i \frac{q}{\hbar} \operatorname{Re}\left\{\left(\int_{-\infty}^{+\infty} \frac{d z^{\prime \prime}}{v_{0}} \tilde{U}\left(z^{\prime \prime}, 0\right) e^{-i \Delta k z^{\prime \prime}}\right) e^{i \Delta k z^{\prime}}\right\} e^{-\frac{\left(z^{\prime}-v_{0} \tau\right)^{2}}{4 v_{0}^{2} \sigma_{p}^{2}}}\right]
\end{aligned}
$$

where the $z^{\prime \prime}$ dependence in the light-profile term is also ignored because $\tilde{U}\left(z^{\prime \prime}\right)$ is only significant in the vicinity of the particle, $z^{\prime \prime} \approx 0$ (see Ref. [31], Appendix, pp. 45-46, for details). We can prove that the $z^{\prime \prime}$ integral in Eq. (7) becomes the field integral $\tilde{F}_{0}$ as

$$
\begin{aligned}
\tilde{F}_{0}(x, y) & \equiv \int_{-\infty}^{+\infty} d z^{\prime \prime} \tilde{E}_{z}\left(x, y, z^{\prime \prime}, 0\right) e^{-i \Delta k z^{\prime \prime}}=\int_{-\infty}^{+\infty} d z^{\prime \prime}\left(-\frac{\partial \tilde{V}_{0}}{\partial z}-\left.\frac{\partial \tilde{A}^{z}}{\partial t}\right|_{t=0}\right) e^{-i \Delta k z^{\prime \prime}} \\
& =\int_{-\infty}^{+\infty} d z^{\prime \prime}\left(-i \Delta k \tilde{V}_{0}+i \omega_{p} \tilde{A}_{0}^{z}\right) e^{-i \Delta k z^{\prime \prime}}=-i \omega_{p} \int_{-\infty}^{+\infty} d z^{\prime \prime}\left(\frac{\tilde{V}_{0}}{v_{0}}-\tilde{A}_{0}^{z}\right) e^{-i \Delta k z^{\prime \prime}}=-i \omega_{p} \int_{-\infty}^{+\infty} d z^{\prime \prime}\left(\frac{\tilde{U}_{0}}{v_{0}}\right) e^{-i \Delta k z^{\prime \prime}}
\end{aligned}
$$

where $\mathbf{E}=-\nabla V-\frac{\partial \mathbf{A}}{\partial t}$ (see Sec. II B) and we utilized $\frac{\partial \tilde{\mathbf{A}}}{\partial t}=-i \omega_{p} \tilde{\mathbf{A}}$ when $\tilde{\mathbf{A}}(t)=\tilde{\mathbf{A}}_{0} e^{-i \omega_{p} t}$ for a single angular frequency [39] and the property of Fourier transform of differentiation, $\mathcal{F}\left\{\left(\frac{d}{d z}\right)^{n} f(z)\right\}=(i k)^{n} \mathcal{F}\{f\}$, for the scalar potential. 

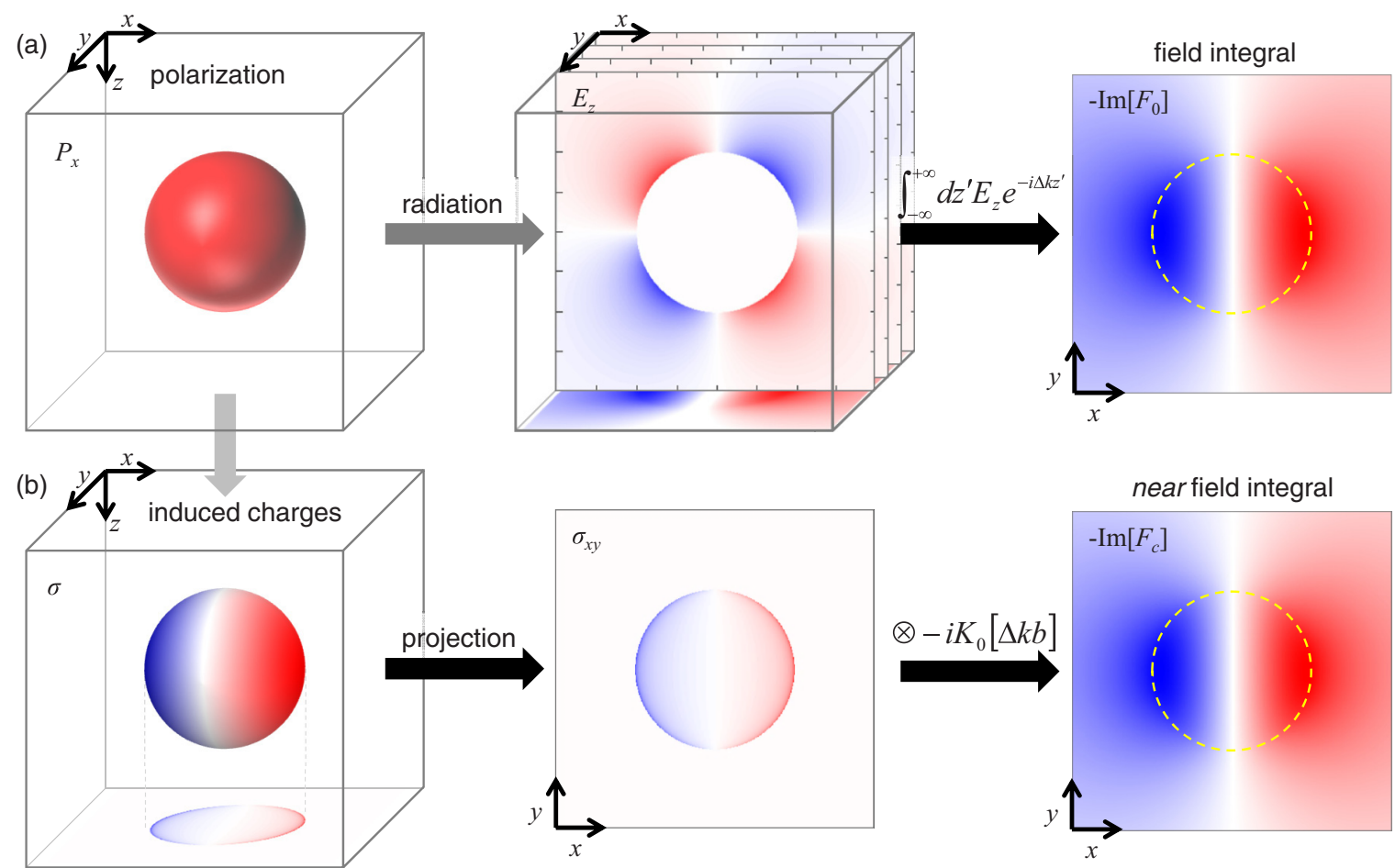

FIG. 1. (Color online) Schemes of evaluating (a) the field integral $\tilde{F}_{0}$ by numerical integration of the electric field and (b) the near-field integral $\tilde{F}_{c}$ by the convolution method. See text for notations.

Therefore, Eq. (7) becomes

$$
\frac{g\left(z^{\prime},+\infty\right)}{g\left(z^{\prime},-\infty\right)}=\exp \left[i \frac{q}{\hbar \omega_{p}} \operatorname{Im}\left\{\tilde{F}_{0} e^{i \Delta k z^{\prime}}\right\} e^{-\frac{\left(z^{\prime}-v_{e} \tau\right)^{2}}{4 v_{e}^{2} \sigma_{p}^{2}}}\right],
$$

and further analysis of this equation follows the methodology given in Ref. [31].

Equation (8) demonstrates that the (electric) field integral, which describes the electron interaction with electromagnetic wave, should incorporate both the scalar and vector potential contributions in general [40]. It also shows that the field integral, which is a Fourier transform of electric field, is also equivalent to a Fourier transform of the sum of scalar and vector potentials (see Sec. III C).

The field integral is determined by the scattered light and the electron velocity, and it describes the degree of photonelectron interaction [31,34]. Therefore, the task of PINEM image simulation reduces to calculating the light scattering of a nanoparticle and then evaluating Eq. (8) in terms of the electric field as a function of the electron trajectory. Figure 1(a) depicts the procedure of evaluating the field integral that was used in previous publications $[31,35,36]$. Once the oscillating polarization $\tilde{\mathbf{P}}$ in the particle is determined, the electric field is calculated by oscillating dipole radiation, and the field integral is evaluated using the first line of Eq. (8).

\section{B. Near field of light scattering}

Describing the excitation of LSP reduces to a light scattering by a nanoparticle, which is typically solved in terms of induced polarization. Fundamentally, polarization is a shift of microscopic charges (electrons with respect to nuclei) [41,42], and the electromagnetic field of an arbitrary, dynamic source can be described by

$$
\mathbf{B}=\nabla \times \mathbf{A}, \quad \mathbf{E}=-\nabla V-\frac{\partial \mathbf{A}}{\partial t} .
$$

In the Lorentz gauge, the retarded potentials are given as

$$
\begin{aligned}
& V(\mathbf{r}, t)=\frac{1}{4 \pi \epsilon_{0}} \iiint d V^{\prime} \frac{\rho\left(\mathbf{r}^{\prime}, t_{r}\right)}{r^{\prime \prime}}, \\
& \mathbf{A}(\mathbf{r}, t)=\frac{\mu_{0}}{4 \pi} \iiint d V^{\prime} \frac{\mathbf{J}\left(\mathbf{r}^{\prime}, t_{r}\right)}{r^{\prime \prime}},
\end{aligned}
$$

where $\rho$ is charge density, $\mathbf{J}$ is current density, $\mathbf{r}^{\prime \prime}=\mathbf{r}-\mathbf{r}^{\prime}$, and $t_{r}=t-\frac{r^{\prime \prime}}{c}$ is retarded time. Note that the surface charge and current densities are implicitly included as the volume quantities. In the Coulomb gauge, the scalar potential is given by instantaneous charges as

$$
V(\mathbf{r}, t)=\frac{1}{4 \pi \epsilon_{0}} \iiint d V^{\prime} \frac{\rho\left(\mathbf{r}^{\prime}, t\right)}{r^{\prime \prime}},
$$

and the retardation effect is incorporated into the vector potential, which is less straightforward to evaluate.

Since it has been demonstrated that the PINEM signal is dominated by the near-field component of scattering [31], we may only consider the near-field term. The scalar potential in the Coulomb gauge provides the simplest (nonretarded) form of the near field of the scattered electric field, where the near field becomes the gradient of a scalar potential only $(\mathbf{E} \approx-\nabla V)$, and it is the form of a Coulomb field of an instantaneous charge density. Namely,

$$
\mathbf{E}(\mathbf{r}, t) \approx \iiint \frac{d V^{\prime} \rho\left(\mathbf{r}^{\prime}, t\right) \hat{\mathbf{r}}^{\prime \prime}}{4 \pi \epsilon_{0}\left|\mathbf{r}^{\prime \prime}\right|^{2}}
$$


For a single frequency of oscillation and by explicitly writing the surface charge densities, (the complex representation of) it becomes

$$
\tilde{\mathbf{E}}(\mathbf{r}, t) \approx\left\{\iiint \frac{d V^{\prime} \rho_{0} \hat{\mathbf{r}}^{\prime \prime}}{4 \pi \epsilon_{0}\left|\mathbf{r}^{\prime \prime}\right|^{2}}+\iint \frac{d A^{\prime} \sigma_{0} \hat{\mathbf{r}}^{\prime \prime}}{4 \pi \epsilon_{0}\left|\mathbf{r}^{\prime \prime}\right|^{2}}\right\} e^{-i \omega_{p} t},
$$

where $\rho\left(\mathbf{r}^{\prime}, t\right)=\operatorname{Re}\left\{\rho_{0}\left(\mathbf{r}^{\prime}\right) e^{-i \omega_{p} t}\right\}$ is the volume charge density and $\sigma\left(\mathbf{r}^{\prime}, t\right)=\operatorname{Re}\left\{\sigma_{0}\left(\mathbf{r}^{\prime}\right) e^{-i \omega_{p} t}\right\}$ is the surface charge density.

\section{Near-field integral}

Expressing the near field via induced charge densities simplifies the calculation of the field integral, and a more direct picture arises as follows: By employing the near field in terms of charge densities, Eq. (14), the field integral, Eq. (8), becomes

$$
\begin{aligned}
\tilde{F}_{c}(x, y)= & \int_{-\infty}^{+\infty} d z\left\{\iiint \frac{d V^{\prime} \rho_{0}}{4 \pi \epsilon_{0}} \frac{\left(z-z^{\prime}\right)}{\left(r^{\prime \prime}\right)^{3}}\right. \\
& \left.+\iint \frac{d A^{\prime} \sigma_{0}}{4 \pi \epsilon_{0}} \frac{\left(z-z^{\prime}\right)}{\left(r^{\prime \prime}\right)^{3}}\right\} \exp [-i \Delta k z] .
\end{aligned}
$$

By performing the $z$ integration first, we obtain the "nearfield integral," which is an approximate field integral with the near-field component only, in terms of the charge density as

$$
\begin{aligned}
\tilde{F}_{c}(x, y)= & \frac{-2 i \Delta k}{4 \pi \epsilon_{0}}\left\{\iiint d V^{\prime} \rho_{0} e^{-i \Delta k z^{\prime}} K_{0}\left[\Delta k b^{\prime \prime}\right]\right. \\
& \left.+\iint d A^{\prime} \sigma_{0} e^{-i \Delta k z^{\prime}} K_{0}\left[\Delta k b^{\prime \prime}\right]\right\} \\
= & -i \frac{\Delta k}{2 \pi \epsilon_{0}} \iint d x^{\prime} d y^{\prime}\left\{\int d z^{\prime} \rho_{0} e^{-i \Delta k z^{\prime}}\right. \\
& \left.+\sum_{z^{\prime} \in A^{\prime}}\left|\frac{\partial^{2} A^{\prime}}{\partial x^{\prime} \partial y^{\prime}}\right| \sigma_{0} e^{-i \Delta k z^{\prime}}\right\} K_{0}\left[\Delta k b^{\prime \prime}\right],
\end{aligned}
$$

where $b^{\prime \prime}=\sqrt{\left(x-x^{\prime}\right)^{2}+\left(y-y^{\prime}\right)^{2}}$ is the impact parameter, $J_{A^{\prime}}^{x y} \equiv\left|\frac{\partial^{2} A^{\prime}}{\partial x^{\prime} \partial y^{\prime}}\right|=\left|\hat{\mathbf{n}}^{\prime} \cdot \hat{\mathbf{z}}^{\prime}\right|^{-1}$ is the Jacobian factor of the particle surface elements to the $x y$ projection, and $K_{n}$ is the modified Bessel function of the second kind. Equation (16) can also be obtained by considering the Coulomb scalar potential, Eq. (12), only in Eq. (8) [43] since the scalar potential describes the near field and the vector potential describes the far field in the Coulomb gauge (see Sec. III C for the significance of the Coulomb potential in the near-field integral).

We note that Eq. (16) is a two-dimensional (2D) convolution of the induced charge density with a broad response function,

$$
\tilde{F}_{c}(x, y)=\left\{\sigma_{x y} \otimes R_{x y}\right\},
$$

where $\sigma_{x y}$ is the projected charge density, given by

$$
\sigma_{x y}\left(x^{\prime}, y^{\prime}\right) \equiv \sum_{z^{\prime} \in A^{\prime}} J_{A^{\prime}}^{x y} \sigma_{0} e^{-i \Delta k z^{\prime}}+\int d z^{\prime} \rho_{0} e^{-i \Delta k z^{\prime}},
$$

and $R_{x y}=\frac{-i \Delta k}{2 \pi \epsilon_{0}} K_{0}[\Delta k b]$ is a response function, with $\otimes$ indicating $2 \mathrm{D}$ convolution. We note that $\sigma_{x y}$ is in the form of a Fourier transform of the induced charge-density distribution within the material, which, in the Rayleigh regime
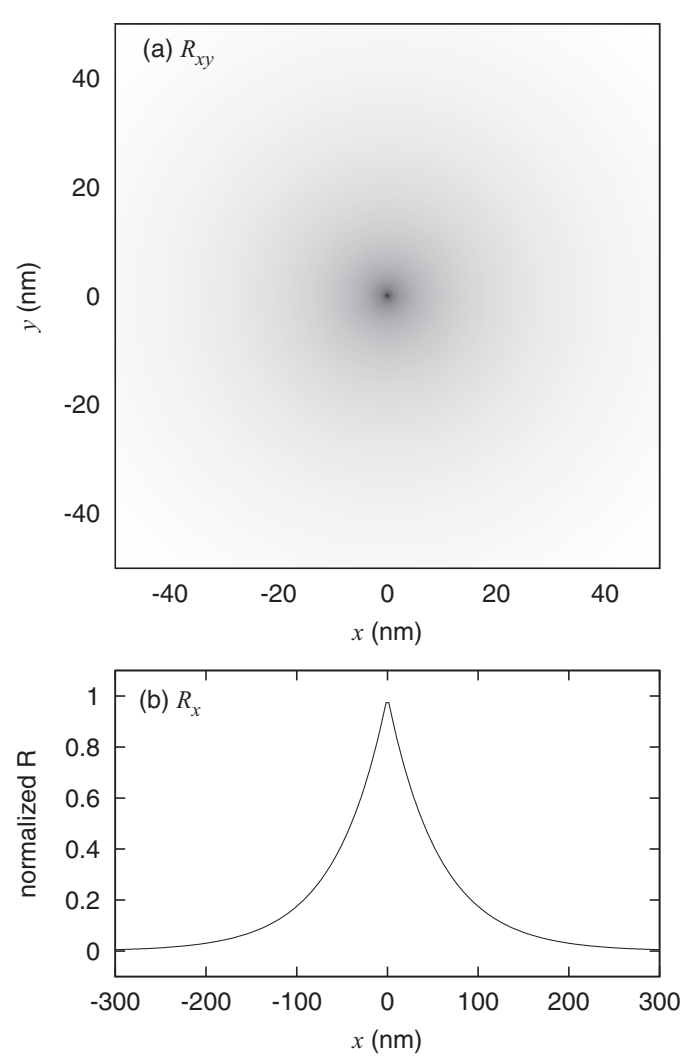

FIG. 2. Profiles of (a) $R_{x y}$ and (b) $R_{x}$ for $\lambda_{p}=519 \mathrm{~nm}$ and $v_{e}=$ $0.695 c$.

$\left(\left|\Delta k z^{\prime}\right| \ll 1\right)$, becomes a simple projection, $\sigma_{x y}\left(x^{\prime}, y^{\prime}\right) \rightarrow$ $\sum_{z^{\prime} \in A^{\prime}} J_{A^{\prime}}^{x y} \sigma_{0}+\int d z^{\prime} \rho_{0}$. For a linear homogeneous material, the induced volume charge density vanishes (see the Appendix), and it further simplifies to a sum of surface charges, $\sigma_{x y}\left(x^{\prime}, y^{\prime}\right) \rightarrow \sum_{z^{\prime} \in A^{\prime}} J_{A^{\prime}}^{x y} \sigma_{0}$. The response function $R_{x y}$ describes the electron interaction with each charge-density oscillation, $\sigma_{x y}\left(x^{\prime}, y^{\prime}, t\right)=\operatorname{Re}\left\{\sigma_{x y}\left(x^{\prime}, y^{\prime}\right) e^{-i \omega_{p} t}\right\}$, as a function of the distance $b$ from the electron trajectory. Note that $K_{0}[\Delta k b]$ is a radially decaying, diffuse function, as shown in Fig. 2(a).

When the charge densities are invariant along the $y$ axis, $\rho_{0}\left(x^{\prime}, y^{\prime}, z^{\prime}\right)=\rho_{0}\left(x^{\prime}, z^{\prime}\right)$ and $\sigma_{0}\left(x^{\prime}, y^{\prime}, z^{\prime}\right)=\sigma_{0}\left(x^{\prime}, z^{\prime}\right)$ (see Secs. III A 2 and III A 3), we can further simplify Eq. (16) by performing the $y^{\prime}$ integration using

$$
\int_{0}^{\infty} d y K_{0}\left[\Delta k \sqrt{x^{2}+y^{2}}\right]=\frac{\pi}{2 \Delta k} \exp [-\Delta k|x|]
$$

[see Sec. 6.677, Eq. (5), in Ref. [44]], and we obtain

$$
\tilde{F}_{c}(x, y)=-\frac{i}{2 \epsilon_{0}} \int d x^{\prime} \sigma_{x y} \exp \left[-\Delta k\left|x^{\prime \prime}\right|\right],
$$

where $x^{\prime \prime}=x-x^{\prime}$. Equation (20) can be alternatively obtained by changing the integration order in Eq. (15), by which we first obtain the near-field component of a line charge density, $E_{z}=\frac{q}{2 \pi \epsilon_{0}} \frac{z}{x^{2}+z^{2}}$, and then its field integral. Similarly, we note that Eq. (20) is a 1D convolution of the induced charge density with an exponential response function,

$$
\tilde{F}_{c}(x, y)=\left\{\sigma_{x y} \otimes R_{x}\right\}
$$


where $R_{x}=-\frac{i}{2 \epsilon_{0}} \exp [-\Delta k|x|]$ [see Fig. 2(b)] and $\otimes$ indicates $1 \mathrm{D}$ convolution in this case. $R_{x}$ describes the electron interaction with a linear charge-density oscillation.

Figure 1(b) depicts procedures of evaluating the near-field integral. Once the oscillating polarization in the particle is determined, induced charge densities are calculated using $\rho=$ $-\nabla \cdot \mathbf{P}$ and $\sigma=\hat{\mathbf{n}} \cdot \mathbf{P}$. Note that the polarization is uniform for the Rayleigh scattering and $\rho$ vanishes. The near-field integral then is obtained from the projected induced charge density convoluted with the $K_{0}$ radial profile using Eq. (17). The field integral $\tilde{F}_{0}$ by a numerical integration and the near-field integral $\tilde{F}_{c}$ show excellent agreement, confirming that the electron-photon interaction is dominated by the near-field component and the retardation effect is negligible.

In this approach, which we call the "convolution method," the electric field in Eq. (8) is further decomposed to the contribution of the charge-density distribution, and physical insight arises therefrom. Namely, Eq. (17) illustrates that the field integral is a direct consequence of the induced charge density of a nanoparticle plasmon via electron-photon interaction. Although the convolution (due to the long-range interaction) broadens the projected charge-density distribution $\sigma_{x y}$ beyond the particle boundary, it retains and visualizes the morphology in $\sigma_{x y}$. Therefore, the multipolar order [45] of a plasmon mode can be directly obtained from PINEM. For example, when a dipole mode prevails such as in Rayleigh scattering, the field integral will show two opposite-sign regions and a node in between. When a quadrupole mode is excited, it will show four alternating-sign regions and nodes among them.

\section{RESULTS AND DISCUSSION}

In this section, we will first apply the convolution method to Rayleigh scattering (dipole approximation) cases for spheres, cylinders, and strips and derive analytical expressions for the field integral in order to demonstrate the validity of the convolution method. Then we utilize the convolution method and investigate the plasmon modes in a silver nanorod in order to illustrate the physical insight made possible by our mathematical treatment of PINEM.

In order to utilize the convolution method, the material polarization in the light-scattering problem needs to be determined first. Light scattering by nanoparticles can be analytically expressed by Mie theory $[46,47]$ for spheres or cylinders of arbitrary sizes and by Rayleigh dipole approximation $[41,48]$ for small ellipsoids. Mie theory has been extended to multilayer spheres [49] and spheres with inhomogeneous refractive indices [50]. For other geometries, numerical methods such as the finite-difference time-domain (FDTD) approach [51], DDA [52,53], or the boundary element method (BEM) $[45,54]$ can be used. For a silver nanorod, we will utilize DDA, which allows one to evaluate the polarizations in the material and hence the induced charge-density distribution. However, the volume charge density should vanish for a linear homogeneous material, and BEM may be invoked to directly evaluate the surface charge densities only [55]. In all cases, the incident light is assumed to be a planar wave propagating in the $+z$ direction with its polarization in the $x$ direction, and substrates are ignored.

\section{A. Dipole cases}

\section{Sphere}

Under Rayleigh approximation, an oscillating uniform polarization is induced in a sphere, resulting in an oscillating, dipolar, surface charge density whose radiation can be treated as an oscillating dipole moment at the center. The near-field approximation of the field integral for a sphere [31] was given as

$$
\tilde{F}_{0} \approx-i \tilde{E}_{0} \cos \phi \chi_{s} \frac{2}{3} a^{3}(\Delta k)^{2} K_{1}[\Delta k b],
$$

where $b=\sqrt{x^{2}+y^{2}}, \cos \phi=\frac{x}{b}$, and $\chi_{s} \equiv \frac{3}{\epsilon+2}(\epsilon-1)$ [56].

Rayleigh dipole approximation for a sphere can be treated as two point charges, $\pm q$, separated by $d$. Equation (16) then becomes

$$
\begin{aligned}
\tilde{F}_{c}= & -i \frac{\Delta k}{2 \pi \epsilon_{0}} \sum_{j} q_{j} e^{-i \Delta k z_{j}^{\prime}} K_{0}\left[\Delta k b_{j}^{\prime \prime}\right] \\
= & -i \frac{\Delta k}{2 \pi \epsilon_{0}}\left\{+q K_{0}\left[\Delta k \sqrt{\left(x-\frac{d}{2}\right)^{2}+y^{2}}\right]\right. \\
& \left.-q K_{0}\left[\Delta k \sqrt{\left(x+\frac{d}{2}\right)^{2}+y^{2}}\right]\right\} \\
\rightarrow & -i \frac{\Delta k}{2 \pi \epsilon_{0}}\left\{q d \Delta k \frac{x}{b} K_{1}[\Delta k b]\right\},
\end{aligned}
$$

where $q d=p_{0}=P_{0} V=\epsilon_{0} \chi_{s} E_{0}\left(\frac{4 \pi}{3} a^{3}\right)$ is the induced dipole moment. Therefore, Eq. (23) reduces to Eq. (22) as $d \rightarrow 0$.

For a uniform polarization, the bulk charge density inside the sphere becomes zero $\left(\rho_{0}=0\right)$, and the surface charge density is given by $\sigma_{0}=P_{0} \cos \theta_{x^{\prime}}=\frac{x^{\prime}}{a} P_{0}$, where $P_{0}=$ $\epsilon_{0} \chi_{s} E_{0}$. The Jacobi factor becomes $J_{A^{\prime}}^{x y}=\left|\frac{a}{z^{\prime}}\right|=\frac{a}{\sqrt{a^{2}-x^{\prime 2}-y^{\prime 2}}}$. Figure 1(b) shows the charge-density distribution, its projection, and the near-field integral. Explicit consideration of the induced charge density in Eq. (17) reproduces the field integral by numerical integration $\left(\tilde{F}_{0}\right)$ in Fig. 1(a), both inside and outside of the sphere projection boundary.

The dipole characteristic of the induced charge-density distribution $\sigma_{x y}$ is directly reflected in the near-field integral $\tilde{F}_{c}$ but broadened by a diffuse profile $R_{x y}$. Even though the field integral is a broadened projection of charge-density distribution, it clearly shows the node because the convolution retains the morphology in $\sigma_{x y}$. Although the PINEM image, which is proportional to $\left|\tilde{F}_{c}\right|^{2}$, loses the phase information, it will show the nodes as intensity minima. We can also see that the (near) field distribution follows the induced charge densities as expected. Once the induced charge-density distribution is obtained, it provides greater insight into the field and the field integral than the polarization itself, which shows a simpler distribution, but its effects are strongly dependent on the geometry (see Sec. III C).

\section{Cylinder}

The near-field approximation of the field integral of cylindrical scattering $[31,35]$ is given by

$$
\tilde{F}_{0} \approx-i \tilde{E}_{0} \cos \phi \chi_{c} \frac{\pi}{2} a^{2} \Delta k \exp [-\Delta k b],
$$


where $\chi_{c} \equiv \frac{2}{\epsilon+1}(\epsilon-1), b=|x|$, and $\phi$ is the incident polarization angle with respect to the cylinder [57].

Dipole approximation for a cylinder can be treated as two line-induced charge densities, $\pm q^{\prime}$, separated by $d$. Equation (20) then becomes

$$
\begin{aligned}
\tilde{F}_{c} & =\frac{-i}{2 \epsilon_{0}} \sum_{j} q_{j}^{\prime} e^{-i \Delta k z_{j}^{\prime}} \exp \left[-\Delta k\left|x_{j}^{\prime \prime}\right|\right] \\
& =\frac{-i}{2 \epsilon_{0}}\left\{q^{\prime} \exp \left[-\Delta k\left|x-\frac{d}{2}\right|\right]-q^{\prime} \exp \left[-\Delta k\left|x+\frac{d}{2}\right|\right]\right\} \\
& \rightarrow \frac{-i}{2 \epsilon_{0}}\left(q^{\prime} d\right) \Delta k \exp [-\Delta k x] \quad\left(\text { for } x>\frac{d}{2}\right),
\end{aligned}
$$

where $q^{\prime} d=p_{0}^{\prime}=P_{0} A=\epsilon_{0} \chi_{c} E_{0}\left(\pi a^{2}\right)$ is the induced dipole moment per length. Therefore, Eq. (25) reduces to Eq. (24) as $d \rightarrow 0$.

For a uniform polarization, the bulk charge density inside the cylinder is zero, and the surface charge density is given by $\sigma_{0}=P_{0} \cos \theta_{x^{\prime}}=\frac{x^{\prime}}{a} P_{0}$, where $P_{0}=\epsilon_{0} \chi_{c} E_{0}$. The Jacobi factor becomes $J_{A^{\prime}}^{x y}=\left|\frac{a}{z^{\prime}}\right|=\frac{a}{\sqrt{a^{2}-x^{\prime 2}}}$. Explicit consideration of the induced charge density in Eq. (20) reproduces a numerically calculated field integral both inside and outside of the cylinder boundary (not shown here).

\section{Strip}

Rayleigh approximation of the field integral for a thin strip [35] of width $w$ and height $h$ was given as

$$
\tilde{F}_{0} \approx-i \tilde{E}_{0} \chi_{b} \frac{h}{2}\left\{e^{-\Delta k\left|x-\frac{w}{2}\right|}-e^{-\Delta k\left|x+\frac{w}{2}\right|}\right\},
$$

where $\chi_{b} \approx \frac{w+h}{w+\epsilon h}(\epsilon-1)$. It can be readily seen that Eq. (26) is Eq. (20) with two line charge densities of $q^{\prime}= \pm \sigma_{0} h$ at the edges, where $\sigma_{0}=P_{0} \approx \epsilon_{0} \chi_{b} E_{0}$.

\section{B. Multipole case}

A silver nanorod of 192-nm length and 20-nm diameter (see Ref. [15] for the geometry) in vacuum was studied using DDA simulations (with 0.5-nm grid size) for optical excitations [36]. Ends were rounded with a radius of curvature of $10 \mathrm{~nm}$. The refractive index of silver was taken from Ref. [58]. It is known that a spheroidal metal nanoparticle exhibits two spectrally separated plasmon resonances, corresponding to oscillations of its conduction electrons along the major or minor axes, respectively [1]. Figure 3 shows DDA-calculated extinction efficiency spectra of the nanorod. Planar wave excitation exhibits a strong anisotropy due to the large aspect ratio of the rod such that the polarization dependence is complementary. The polarization parallel to the axis shows resonances at lower photon energies (longer wavelengths) compared to the perpendicular polarization, which only shows a broad resonance: resonances at 1130, 489, and $400 \mathrm{~nm}$ for the parallel polarization and at $342 \mathrm{~nm}$ for the perpendicular polarization with respect to the rod axis.

Figure 4 shows scattered fields, polarizations, induced charge densities, and near-field integrals at the first three resonance wavelengths for parallel $(\hat{\mathbf{x}})$ polarization along the rod axis. The polarizations in a nanorod at resonances can be well described by standing waves [26]. The polarizations in

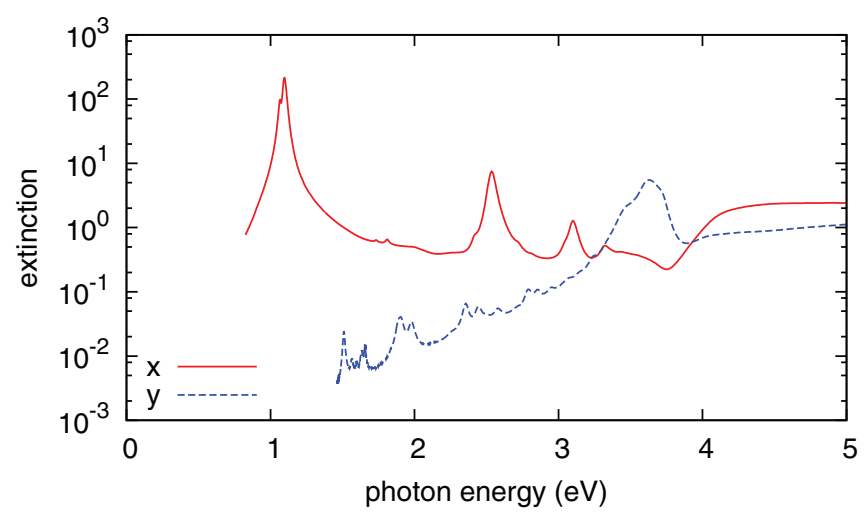

FIG. 3. (Color online) Optical extinction efficiencies of a silver nanorod at parallel $(\hat{x}$, solid red line) and perpendicular $(\hat{y}$, dashed blue line) polarizations.

Fig. 4 show one, three, and five extrema at the first, second, and third resonance energies, respectively. However, the electricfield magnitude outside the rod is strong at two, four, and six regions, respectively, whereas the electric field inside the rod follows the polarization. Furthermore, the simulated PINEM images also show two, four, and six maxima along the rod, illustrated by extrema in the near-field integral. The calculated charge density (see the Appendix) reveals the mechanism behind those observations. Those observations result from the distribution of the charge density, the morphology of which is directly reflected in the field integral. Namely, the charge-density distribution explains the distributions of the field integral as well as the electric field.

Plasmon modes in Fig. 4 also agree with those in silver nanorods $[15,26]$ and a nanocarrot [59] determined by STEMEELS experiments. Their STEM-EELS images can be also understood with the theory presented in this paper, and the induced charge-density picture here clearly reveals the spatial distributions of plasmon modes at resonances.

\section{Correlations}

As shown in this paper, the electric field and the field integral are directly related to the charge-density distribution. Equation (17) shows that the near-field integral is a convolution of the projected charge density and a $2 \mathrm{D}$ radial response function which monotonically decreases as a function of the impact parameter $b$. Note that the modified Bessel function can be approximated as $K_{0}(z) \approx-\ln \frac{z}{2}-\gamma$ for $z \ll 1$, where $\gamma$ is the Euler-Mascheroni constant. Similarly, the Coulomb potential, Eq. (12), can be seen as a convolution of the charge density and a 3D radial response function which is a reciprocal function of the distance $r$. If we ignore the $z$ coordinates of the charge densities, we can approximate the Coulomb potential at $z=0$ as a $2 \mathrm{D}$ convolution, $V(x, y, 0) \approx$ $\frac{1}{4 \pi \epsilon_{0} b} \otimes \sigma_{x y}$, which is in a form similar to the near-field integral. The similarity is further evident from the fact that $K_{0}$ in the near-field integral equivalently results from Fourier transform of the Coulomb potential because the near-field approximation of Eq. (8) becomes a Fourier transform of $\tilde{V}$ only in $\tilde{U}$. The electric-field $z$ component above the particle $E_{z}(x, y, h)$, where $h$ is comparable to the particle height, is 


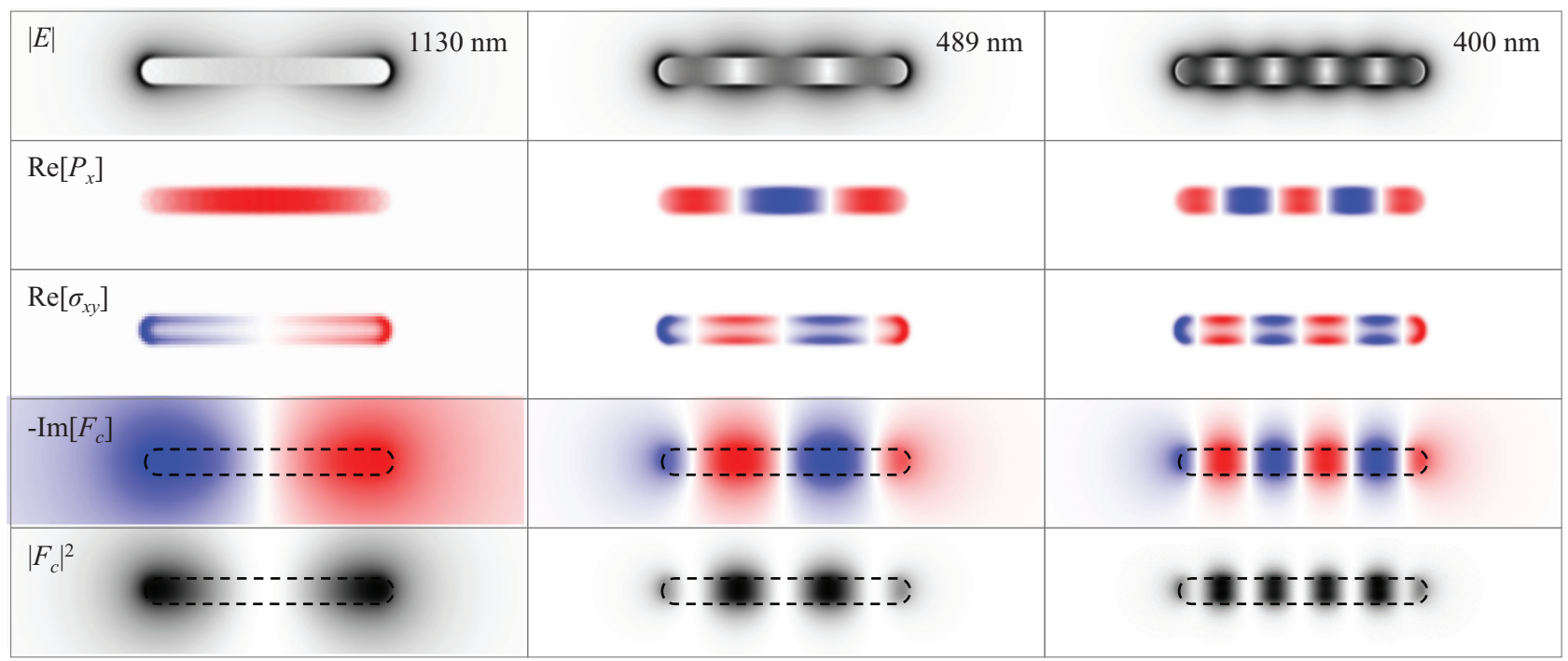

FIG. 4. (Color online) Scattered field $E$ at $z=0$ (the center of the rod), polarization $P$ at $z=0$, projected charge density $\sigma_{x y}$, near-field integral $\tilde{F}_{c}$, and the PINEM image $\left|\tilde{F}_{c}\right|^{2}$ of a silver nanorod for $x$-polarized incident light at 1130,489 , and $400 \mathrm{~nm}$.

often invoked to explain STEM-EELS images because it is related to the maximum contribution to the field integral, whereas $E_{z}(x, y, 0)$ becomes zero for symmetric particles. Similar to the Coulomb potential, ignoring the $z$ coordinates of the charge densities, it can be approximated as a $2 \mathrm{D}$ convolution, $E_{z}(x, y, h) \approx \frac{h}{4 \pi \epsilon_{0} \sqrt{h^{2}+h^{2}}} \otimes \sigma_{x y}$. Therefore, $\tilde{F}_{c}$, $V$, and $E_{z}$ are correlated to each other, and they are related to $\sigma_{x y}$, exactly or approximately.

The similarities of those quantities to the field integral are most apparent in the case of the field enhancement between two scattering centers. Figure 5 shows $\sigma_{x y}, \tilde{F}_{c}, V(x, y, 0)$, $E_{z}(x, y, h)$, and $E_{x}(x, y, 0)$ for two weakly scattering dielectric spheres, separated by the distance of one radius at an angle of $15^{\circ}$ with respect to the incident light polarization $(\hat{\mathbf{x}})$. Note that the potential and near field of each sphere are given by the Coulomb potential and field of a dipole, multiplied by a temporal oscillation [41]. An aligned dimer can constructively increase the scattered field between the particles, as shown in the bottom panel of Fig. 5. However, $\tilde{F}_{c}(x, y), V(x, y, 0)$, and $E_{z}(x, y, h)$ all show destructive interference between the particles. In particular, the Coulomb potential shows a fairly good correlation to the field integral, except that it exhibits a shorter decay than the field integral. $E_{z}(x, y, h)$ shows a feature that is correlated but less similar to that of the field integral. Figure 5 demonstrates that the field integral cannot be directly compared to the field strength $|\mathbf{E}(x, y, 0)|$, especially for the junction field which is dominated by the $E_{x}$ component, between particles $[25,38]$ but should be interpreted as a quantity correlated to the Coulomb potential, the gradient of which gives the near field. Therefore, the scattering field and intensity are correlated to the slope of (the square root of) the PINEM intensity, not its absolute value. Consequently, nodes (absence of signal) in PINEM images correspond to the strong electric field.

\section{SUMMARY}

The near-field approximation allows one to describe the scattered electric field in the vicinity of nanoparticles as the
Coulomb field of instantaneous charges and, consequently, to relate the electric field and the field integral to the induced charge density in nanoparticles. The physical meaning of the field integral in the PINEM theoretical formulation was reexamined in terms of the scattering near field, and it was found that the near-field integral becomes the $2 \mathrm{D}$ projection of the optically driven charge-density distribution convoluted with a diffuse response function. The response function is given by a Bessel function or by an exponential function with an argument of $\Delta k b$, and hence, the effective range is on the order of $\Delta k^{-1}=\frac{\lambda}{2 \pi} \frac{v_{e}}{c}=\sim 60 \mathrm{~nm}$ for 519-nm incident light and a $200-\mathrm{keV}$ electron. Physically, PINEM measures the electron interaction with charge-density oscillations via $E_{z}$, and its interaction is simply given by those response functions. Therefore, PINEM (field integral) is mathematically related to the charge-density projection and can be used to investigate the induced charge-density distribution of the plasmon modes in nanoparticles.

The projected charge-density convolution method was first applied to dipole cases, and it was shown that the previous results of analytical expressions for the field integrals for spheres, cylinders, and strips are reproduced in the region outside of the particle boundaries by the convolution method. Furthermore, the numerically calculated field integrals are also reproduced by the convolution method both inside and outside of the particle boundaries when the induced charge-density distributions are explicitly considered. The convolution method was then applied to a silver nanorod to demonstrate that PINEM visualizes the plasmon modes under optical excitation, and it was found that multipoles can be excited along the axis in a finite rod and the field integrals clearly reflect the multipolar order in particles.

The physical insight gained from our formulation of the field integral greatly simplifies the interpretation of PINEM images. Namely, plasmons are collective oscillations of the free electrons in metal, and PINEM is directly related to the optically driven induced charge densities. The projected charge density and the near-field integral were compared to other 


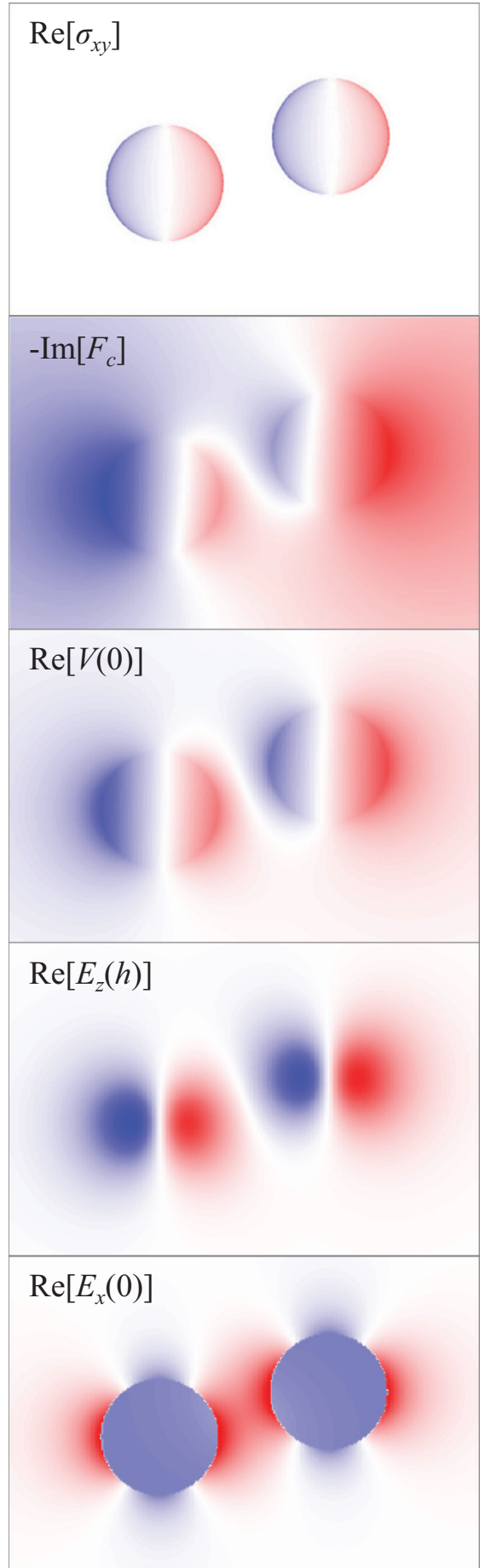

FIG. 5. (Color online) Projected charge density $\sigma_{x y}$, near-field integral $\tilde{F}_{c}$, Coulomb potential $V$ at $z=0$, and scattered electric fields $\left(E_{z}\right.$ at $z=h$ and $E_{x}$ at $z=0$ ) of a dimer of small (10-nm radius) spheres. Note that $h$ was chosen to be one radius here.

relevant physical quantities, such as Coulomb potential and electric fields. It was found that the Coulomb potential is well correlated with the near-field integral because the near-field integral, which is a Fourier transform of the field, is also a Fourier transform of the scalar potential in the near-field approximation. However, the electric field, in particular the junction field, may not correlate with the PINEM intensity itself.

In principle, the convolution method developed here is independent of the plasmon excitation mechanism and therefore is also applicable to EELS [33]. Namely, the EELS signal at the electron trajectory of $(x, y)$ is a sum of the oscillating charge density induced by a traveling electron, weighted by $K_{0}[\Delta k b]$. However, at different positions of the electron, the induced charge-density distribution changes, and therefore, it is not a map of a single-plasmon mode but a map of electric excitation efficiency. In fact, the excitation profile is also given by Bessel functions [45], and EELS intensity can be expressed as an overlap among the excitation profile, plasmon field, and probing profile, where the excitation is given by $K_{0}$ and $K_{1}$ and the probing is given by $K_{0}$. The optical excitation mechanism of PINEM enables visualization of the plasmon in optical excitation, while EELS only produces an efficiency map of electric excitation.

\section{ACKNOWLEDGMENTS}

This work was supported by the National Science Foundation and the Air Force Office of Scientific Research in the Center for Physical Biology funded by the Gordon and Betty Moore Foundation. The authors wish to thank the referees for the thorough review and helpful comments. S.T.P. would like to thank Dr. Giovanni M. Vanacore, Dr. Timothy D. Scarborough, and Dr. J. Spencer Baskin for fruitful discussions.

\section{APPENDIX: STANDING-WAVE FORMULATION}

The polarization in the silver nanorod can be represented by a standing wave as

$$
P_{x}(x)=P_{0} \sin \frac{n \pi x}{L}
$$

for $x=[0, L]$. Since the volume charge density vanishes in a linear homogeneous material $\left(\rho=-\nabla \cdot \mathbf{P}\right.$, where $\mathbf{P}=\chi \epsilon_{0} \mathbf{E}$, $\mathbf{D}=\epsilon \epsilon_{0} \mathbf{E}$, and no explicit charge, $\left.0=\rho_{e x}=\nabla \cdot \mathbf{D}\right)$, we expect, for a cylindrically symmetric geometry along the $x$ axis,

$$
0=\frac{\partial P_{x}}{\partial x}+\frac{\partial\left(\zeta P_{\zeta}\right)}{\zeta \partial \zeta}
$$

where $\zeta=\sqrt{y^{2}+z^{2}}$. Then we obtain

$$
\frac{\partial\left(\zeta P_{\zeta}\right)}{\zeta \partial \zeta}=-\frac{\partial P_{x}}{\partial x}=Q_{0} \cos \frac{n \pi x}{L},
$$

where $Q_{0}=-\frac{n \pi P_{0}}{L}$, and therefore,

$$
P_{\zeta}(x, \zeta)=Q_{0} \frac{\zeta}{2} \cos \frac{n \pi x}{L} .
$$

The surface charge on the side becomes

$$
\sigma(x)=\hat{\zeta} \cdot \mathbf{P}=P_{\zeta}(x, a)=Q_{0} \frac{a}{2} \cos \frac{n \pi x}{L},
$$

where $a$ is the rod radius. Note that the optical resonances shown in Fig. 4 correspond to $n=1,3$, and 5, respectively, and the polarization, Eq. (A1), has $n$ extrema, whereas the surface charge, Eq. (A5), has $n+1$ extrema and $n$ nodes along the $x$ direction. 
[1] S. A. Maier, Plasmonics: Fundamentals and Applications (Springer, New York, 2007).

[2] J. M. Pitarke, V. M. Silkin, E. V. Chulkov, and P. M. Echenique, Rep. Prog. Phys. 70, 1 (2007).

[3] J. Zhang, L. Zhang, and W. Xu, J. Phys. D 45, 113001 (2012).

[4] S. M. Nie and S. R. Emery, Science 275, 1102 (1997).

[5] G. Haran, Acc. Chem. Res. 43, 1135 (2010).

[6] J. Nelayah, M. Kociak, O. Stéphan, F. J. García de Abajo, M. Tencé, L. Henrard, D. Taverna, I. Pastoriza-Santos, L. M. Liz-Marzán, and C. Colliex, Nat. Phys. 3, 348 (2007).

[7] J. Nelayah, M. Kociak, O. Stephan, N. Geuquet, L. Henrard, F. J. García de Abajo, I. Pastoriza-Santos, L. M. Liz-Marzan, and C. Colliex, Nano Lett. 10, 902 (2010).

[8] J. Nelayah, I. Gu, W. Sigle, C. T. Koch, I. Pastoriza-Santos, L. M. Liz-Marzán, and P. A. van Aken, Opt. Lett. 34, 1003 (2009).

[9] M. Rang, A. C. Jones, F. Zhou, Z. Y. Li, B. J. Wiley, Y. N. Xia, and M. B. Raschke, Nano Lett. 8, 3357 (2008).

[10] B. Schaffer, U. Hohenester, A. Trugler, and F. Hofer, Phys. Rev. B 79, 041401 (2009).

[11] W. Sigle, J. Nelayah, C. T. Koch, and P. A. van Aken, Opt. Lett. 34, 2150 (2009).

[12] I. Arslan, J. K. Hyun, R. Erni, M. N. Fairchild, S. D. Hersee, and D. A. Muller, Nano Lett. 9, 4073 (2009).

[13] U. Hohenester, H. Ditlbacher, and J. R. Krenn, Phys. Rev. Lett. 103, 106801 (2009).

[14] A. L. Koh, K. Bao, I. Khan, W. E. Smith, G. Kothleitner, P. Nordlander, S. A. Maier, and D. W. McComb, ACS Nano 3, 3015 (2009).

[15] B. S. Guiton, V. Iberi, S. Z. Li, D. N. Leonard, C. M. Parish, P. G. Kotula, M. Varela, G. C. Schatz, S. J. Pennycook, and J. P. Camden, Nano Lett. 11, 3482 (2011).

[16] I. Alber, W. Sigle, S. Muller, R. Neumann, O. Picht, M. Rauber, P. A. van Aken, and M. E. Toimil-Molares, ACS Nano 5, 9845 (2011).

[17] A. I. Henry, J. M. Bingham, E. Ringe, L. D. Marks, G. C. Schatz, and R. P. Van Duyne, J. Phys. Chem. C 115, 9291 (2011).

[18] K. Andersen, K. W. Jacobsen, and K. S. Thygesen, Phys. Rev. B 86, 245129 (2012).

[19] M. G. Blaber, A. I. Henry, J. M. Bingham, G. C. Schatz, and R. P. Van Duyne, J.Phys. Chem. C 116, 393 (2012).

[20] H. G. Duan, A. I. Fernandez-Dominguez, M. Bosman, S. A. Maier, and J. K. W. Yang, Nano Lett. 12, 1683 (2012).

[21] P. R. Edwards, L. K. Jagadamma, J. Bruckbauer, C. W. Liu, P. Shields, D. Allsopp, T. Wang, and R. W. Martin, Microsc. Microanal. 18, 1212 (2012).

[22] S. Mazzucco, N. Geuquet, J. Ye, O. Stephan, W. Van Roy, P. Van Dorpe, L. Henrard, and M. Kociak, Nano Lett. 12, 1288 (2012).

[23] V. Myroshnychenko, J. Nelayah, G. Adamo, N. Geuquet, J. Rodriguez-Fernandez, I. Pastoriza-Santos, K. F. MacDonald, L. Henrard, L. M. Liz-Marzan, N. I. Zheludev, M. Kociak, and F. J. García de Abajo, Nano Lett. 12, 4172 (2012).
[24] N. W. Bigelow, A. Vaschillo, V. Iberi, J. P. Camden, and D. J. Masiello, ACS Nano 6, 7497 (2012).

[25] N. Mirsaleh-Kohan, V. Iberi, P. D. Simmons, N. W. Bigelow, A. Vaschillo, M. M. Rowland, M. D. Best, S. J. Pennycook, D. J. Masiello, B. S. Guiton, and J. P. Camden, J. Phys. Chem. Lett. 3, 2303 (2012).

[26] D. Rossouw, M. Couillard, J. Vickery, E. Kumacheva, and G. A. Botton, Nano Lett. 11, 1499 (2011).

[27] F. J. García de Abajo and M. Kociak, New J. Phys. 10, 073035 (2008).

[28] B. Barwick, D. J. Flannigan, and A. H. Zewail, Nature (London) 462, 902 (2009).

[29] D. J. Flannigan, B. Barwick, and A. H. Zewail, Proc. Natl. Acad. Sci. USA 107, 9933 (2010).

[30] F. J. García de Abajo, A. Asenjo-Garcia, and M. Kocíak, Nano Lett. 10, 1859 (2010).

[31] S. T. Park, M. Lin, and A. H. Zewail, New J. Phys. 12, 123028 (2010).

[32] A. Howie, Eur. Phys. J. Appl. Phys. 54, 33502 (2011).

[33] A. Asenjo-Garcia and F. J. García de Abajo, New J. Phys. 15, 103021 (2013).

[34] S. T. Park and A. H. Zewail, J. Phys. Chem. A 116, 11128 (2012).

[35] S. T. Park, A. Yurtsever, J. S. Baskin, and A. H. Zewail, Proc. Natl. Acad. Sci. USA 110, 9277 (2013).

[36] S. T. Park and A. H. Zewail, Proc. SPIE 8845, 884506 (2013).

[37] A. Yurtserver, R. M. van der Veen, and A. H. Zewail, Science 335, 59 (2012).

[38] A. Yurtserver, J. S. Baskin, and A. H. Zewail, Nano Lett. 12, 5027 (2012).

[39] Note that the slowly varying envelope function has been factored out in Eq. (6).

[40] Previously, in Refs. [31] and [34], the field integral was defined in terms of the electric field, assuming $\mathbf{E}=-\frac{\partial \mathbf{A}}{\partial t}$ and $V=0$, which is a special case of Eq. (8) in this paper, namely, the far field in the Coulomb gauge.

[41] J. D. Jackson, Classical Electrodynamics (Wiley, New York, 1975).

[42] D. J. Griffiths, Introduction to Electrodynamics (Prentice Hall, Upper Saddle River, NJ, 1999).

[43] Indeed, we obtain $\int_{-\infty}^{+\infty} \frac{1}{r} e^{-i \Delta k z}=2 K_{0}[\Delta k b]$, where $r=$ $\sqrt{b^{2}+z^{2}}$, for the Coulomb potential contribution in the third line of Eq. (8).

[44] I. S. Gradshteyn and I. M. Ryzhik, Table of Integrals, Series, and Products, 4th ed. (Academic, New York, 1965).

[45] F. J. García de Abajo, Rev. Mod. Phys. 82, 209 (2010).

[46] G. Mie, Ann. Phys. (Berlin) 25, 377 (1908).

[47] H. C. van der Hulst, Light Scattering by Small Particles (Wiley, New York, 1957).

[48] R. Gans, Ann. Phys. (Lepzig) 37, 881 (1912).

[49] B. R. Johnson, Appl. Opt. 35, 3286 (1996).

[50] A. Y. Perelman, Appl. Opt. 35, 5452 (1996).

[51] R. Courant, K. O. Friedrichs, and H. Lewy, Math. Ann. 100, 32 (1928).

[52] E. M. Purcell and C. R. Pennypacker, Astrophys. J. 186, 705 (1988).

[53] B. T. Draine, Astrophys. J. 333, 848 (1988). 
[54] F. J. García de Abajo and A. Howie, Phys. Rev. B 65, 115418 (2002).

[55] BEM directly calculates the self-consistent induced surface charge density that satisfies the boundary conditions with an external field.

[56] Note that $\chi_{s}$ is defined to include a factor of 3 here, different from Ref. [31].
[57] Note that $\chi_{c}$ is defined to include a factor of 2 here (see Ref. [35]), different from Ref. [31].

[58] P. B. Johnson and R. W. Christy, Phys. Rev. B 6, 4370 (1972).

[59] H. Y. Liang, D. Rossouw, H. G. Zhao, S. K. Cushing, H. L. Shi, A. Korinek, H. X. Xu, F. Rosei, W. Z. Wang, N. Q. Wu, G. A. Botton, and D. L. Ma, J. Am. Chem. Soc. 135, 9616 (2013). 